\title{
Total Peritoneal Gutter Removal versus Random Peritoneal Biopsy in Cases of Ovarian Cancer
}

\author{
Ahmed Abdel-Azeem Essmat', Mahmoud El-Sayed Meleis' ${ }^{1}$, Helmy Abdel-Sattar Rady', \\ Inass Ibrahim Ahmed Zaki ${ }^{2}$, Ahmed Shaaban Ali Ragab Khattab ${ }^{1}$
}

${ }^{1}$ Department of Obstetrics and Gynecology, Faculty of Medicine, Alexandria University, Alexandria, Egypt

${ }^{2}$ Department of Pathology, Faculty of Medicine, Alexandria University, Alexandria, Egypt

Email:princeessmat@gmail.com

How to cite this paper: Essmat, A.A.-A., Meleis, M.E.-S., Rady, H.A.-S., Zaki, I.I.A. and Khattab, A.S.A.R. (2021) Total Peritoneal Gutter Removal versus Random Peritoneal Biopsy in Cases of Ovarian Cancer. Open Journal of Obstetrics and Gynecology, 11, 1342-1350.

https://doi.org/10.4236/ojog.2021.1110125

Received: July 16, 2021

Accepted: October 16, 2021

Published: October 19, 2021

Copyright $\odot 2021$ by author(s) and Scientific Research Publishing Inc. This work is licensed under the Creative Commons Attribution International License (CC BY 4.0).

http://creativecommons.org/licenses/by/4.0/

(c) (i) Open Access

\begin{abstract}
Introduction: Ovarian cancer is the fourth most prevalent cancer among women and the seventh most common cancer overall. Every year, an estimated 200,000 cases and 125,000 deaths related to ovarian cancer are reported around the world. It is most common in high-resource countries, with an incidence rate of 9.3 per 100,000 women. Ovarian cancer is detected at an advanced stage in about $70 \%$ of instances, and only $30 \%$ of women with such cancers live for more than 5 years. Although only around $20 \%$ of ovarian cancers are limited to the ovaries at diagnosis, patients with localized disease have a 5 -year survival rate of more than $90 \%$. Peritonectomy is a crucial part of the surgical treatment for ovarian cancer. Visual inspection and palpation are not reliable methods for determining the extent of tumour involvement. The majority of gynecologic oncologists are suspicious about the benefits of a full peritonectomy, concerned about the benefits and hazards. They believe that the tumor's fundamental biology, not surgical aggressiveness, dictates the tumor's resectability. The aim of this work was primarily to compare the differences between total peritoneal gutter removal and random peritoneal biopsy in cases of early ovarian cancer. A secondary aim of this work is to show ability of total peritoneal gutter removal in relation to that of random peritoneal biopsy to detect positivity of metastasis in the histopathological specimens. Patients and Methods: This prospective cohort study was conducted on 130 patients with early ovarian cancer in El-Shatby hospital, Faculty of Medicine, Alexandria University. For each patient, we took random and total peritoneal biopsy and compared between them regarding technique, timing, post-operative complications, and histopathological results. Inclusion criteria: all age group, suspected ovarian cancer using IOTA score, absence of nodules
\end{abstract}


in the peritoneal gutter using CT and any case that needs staging laparotomy for ovarian cancer. Exclusion criteria: presence of gross peritoneal disease in the gutter and CT features of advanced ovarian cancer (peritoneal deposits, omental cakes, pelvic and para-aortic lymphadenopathy). All cases will be subjected to the followings: history taking, clinical examination, suspicious ovarian mass using IOTA score, tumor markers, CT staging, staging laparotomy and random and total peritoneal biopsies will be collected from the same case and sent to the lab of pathology for histopathological examination and the results will be compred regarding positivity of metastasis. Results: Random peritoneal biopsy showed positive metastasis in $6(4.6 \%)$ cases and free in 124 (95.4\%) cases while total peritoneal gutter removal showed positive metastasis in $19(14.6 \%)$ cases and free in $111(85.4 \%)$ with Chi-square test was 7.479 and $\mathrm{P}$ value was 0.004 which is statistically significant. 108 (97.3\%) cases had negative random peritoneal biopsy and negative total peritoneal gutter removal, $3(2.7 \%)$ cases had positive random peritoneal biopsy and negative total peritoneal gutter removal, $16(84.2 \%)$ cases had positive total peritoneal gutter removal and negative random peritoneal biopsy \& 3 (15.8\%) cases had positive total peritoneal gutter removal and positive random peritoneal biopsy with Chi-square test $6.311,{ }^{\mathrm{FE}} \mathrm{p} 0.04$ and Kappa test $0.183(0.012)$ which is statistically significant with poor strength of agreement. Conclusions: Modified total peritoneal gutter removal is a safe, fast and easy technique compared to random peritoneal biopsy and even to traditional peritonectomy procedure with high detection rates of peritoneal involvement with sensitivity of $50 \%$ and specificity of $87.5 \%$ and it is recommended to be done as a routine alternative to random peritoneal biopsy and even to traditional total gutter removal in all cases of ovarian malignancies for diagnostic and therapeutic values.

\section{Keywords}

Pelvic Peritonectomy, Gutter Resection, Ovarian Cancer

\section{Introduction}

Ovarian cancer is the fourth most prevalent cancer among women and the seventh most common cancer overall. Every year, an estimated 200,000 cases and 125,000 deaths related to ovarian cancer are reported around the world. It is most common in high-resource countries, with an incidence rate of 9.3 per 100,000 women [1].

Solid tumours with thick capsules and papillay projections on ultrasonography are extremely suspected of being malignant. CT staging is used to assess whether or not the cancer has spread to the liver, lung, lymph nodes, peritoneal surfaces, or omentum. If the lesion is considered to be of mesenteric origin, an MRI pelvis may be used to show the anatomical origin of the lesion. Fine needle aspiration or core biopsy can be used to obtain a biopsy of the tumour. The as- 
cetic fluid is examined. In the majority of cases, CA 125 is measured and found to be increased. CEA levels are elevated in GIT malignancies. AFP and BHCG are essential in malignancies in women under the age of 40 , as this group is more likely to develop germ cell tumours. Other tests may include a complete blood count, as well as liver and kidney function tests [2].

Surgical staging begins with an infraumbilical midline incision that can be extended above the umbilicus, followed by aspiration of ascetic fluid or collection of peritoneal washings. Then all peritoneal surfaces are examined and palpated. The procedure includes a total abdominal hysterectomy and BSO. The infracolic omentum should be removed if there is no substantial extra-ovarian illness. Also collected are random peritoneal biopsies or scrapings, ideally around the diaphragms. A pelvic and infrarenal paraaortic lymphadenectomy, the most important stage, is also accomplished [3].

Peritonectomy is a crucial part of the surgical treatment for ovarian cancer. Visual inspection and palpation are not reliable methods for determining the extent of tumour involvement. The majority of gynecologic oncologists are suspicious about the benefits of a full peritonectomy, concerned about the benefits and hazards. They believe that the tumor's fundamental biology, not surgical aggressiveness, dictates the tumor's resectability [4].

Peritoneal implants are divided into invasive and non-invasive categories, with non-invasive implants further subdivided into epithelial, desmoplastic, or both [5].

Invasive peritoneal implants have different criteria than non-invasive serous ovarian cancers because they affect prognosis and treatment outcomes. Based on the histopathologic appearance, peritoneal implants were classed as either noninvasive or invasive. Invasion of underlying normal tissue, micropapillary architecture, and solid epithelial nests surrounded by clefts were shown as three diagnostic criteria for invasive implants [6].

Non-invasive peritoneal implants may have a papillary core with psammoma bodies lined by epithelium and weak adhesion to neighbouring fibrofatty tissue without invading the surrounding structures when viewed under a microscope. Invasive peritoneal implants, on the other hand, may exhibit a papillary core and solid epithelial nest encircled by a cleft penetrating the underlying adipose tissue [7].

\section{Patients and Methods}

This prospective cohort study was conducted on 130 patients with early ovarian cancer in El-Shatby hospital, Faculty of Medicine, Alexandria University.

For each patient, we took random and total peritoneal biopsy and compared between them regarding technique, timing, post-operative complications, and histopathological results. All cases will be subjected to the followings: history taking regarding age, marital status, gravidity, parity, abortion, menopausal state and family history of cancer, clinical examination regarding ascites, consistency, 
mobility, tenderness and surface of the ovarian mass, suspicious ovarian mass using IOTA score regarding laterality, simple rules of IOTA and IOTA ADNEX model. We used Mindray DC-70 ultrasound system both transvaginal and transabdominal probes, tumor markers: CA 125, CEA, CA 19.9 \& CA 15.3 for patients older than 40 years old and AFP, LDH \& BHCG for those younger than this age, CT staging to exclude liver metastasis, lung metastasis, lymph node affection and ascites, staging laparotomy will be performed in cases of ovarian cancer, random peritoneal biopsy is taken by grasping a snip of peritoneum at the paracolic gutter at one side and cut by coagulation diathermy or better by a scalpel in order not to damage the margins of the biopsy, paracolic peritoneal gutter, at the other side, is totally excised by cutting at the white line to allow colon mobilization from below upwards till we reach to phrenicocolic ligament on left side or hepatic flexure on right side. After complete mobilization of the colon, dissection of peritoneum is continued from medial to lateral till we reach midline incision. Instead of extending incision to the upper region of paracolic gutters, we removed a rectangular area of parietal peritoneum that extends from back of iliac crest inferiorly to peritoneal reflection at level of transverse colon superiorly and to back of rectus sheath laterally. This technique is referred to as modified peritoneal gutter resection technique. This procedure is done using cold knife, then combined sharp and blunt dissection to separate peritoneum from underlying tissue and finally random peritoneal biopsy and total peritoneal gutter were sent to Lab of Pathology at Faculty of Medicine, Alexandria University and were compared regarding positivity of metastasis.

\section{Results}

\section{Sociodemographic and clinical characteristics}

Random peritoneal biopsy showed positive metastasis in $6(4.6 \%)$ cases and free in 124 (95.4\%) cases while total peritoneal gutter removal showed positive metastasis in $19(14.6 \%)$ cases and was free in 111 (85.4\%) with Chi-square test was 7.479 and $\mathrm{P}$ value was 0.004 which is statistically significant. 108 (97.3\%) cases had negative random peritoneal biopsy and negative total peritoneal gutter removal, 3 (2.7\%) cases had positive random peritoneal biopsy and negative total peritoneal gutter removal, $16(84.2 \%)$ cases had positive total peritoneal gutter removal and negative random peritoneal biopsy \& 3 (15.8\%) cases had positive total peritoneal gutter removal and positive random peritoneal biopsy with Chi-square test $6.311,{ }^{\mathrm{FE}} \mathrm{p} 0.04$ and Kappa test 0.183 (0.012) which is statistically significant with poor strength of agreement.

In comparison of total peritoneal gutter removal to random peritoneal biopsy, the results showed sensitivity $15.79 \%$, specificity $97.3 \%$, positive predictive value $50 \%$, negative predictive value $87.1 \%$ with accuracy $85.38 \%$. On the other side, when we compared random peritoneal biopsy to total peritoneal gutter removal, we found that sensitivity was $50 \%$, specificity was $78.1 \%$, positive predictive value was $15.79 \%$, negative predictive value was $97.3 \%$ and accuracy was $85.38 \%$. 


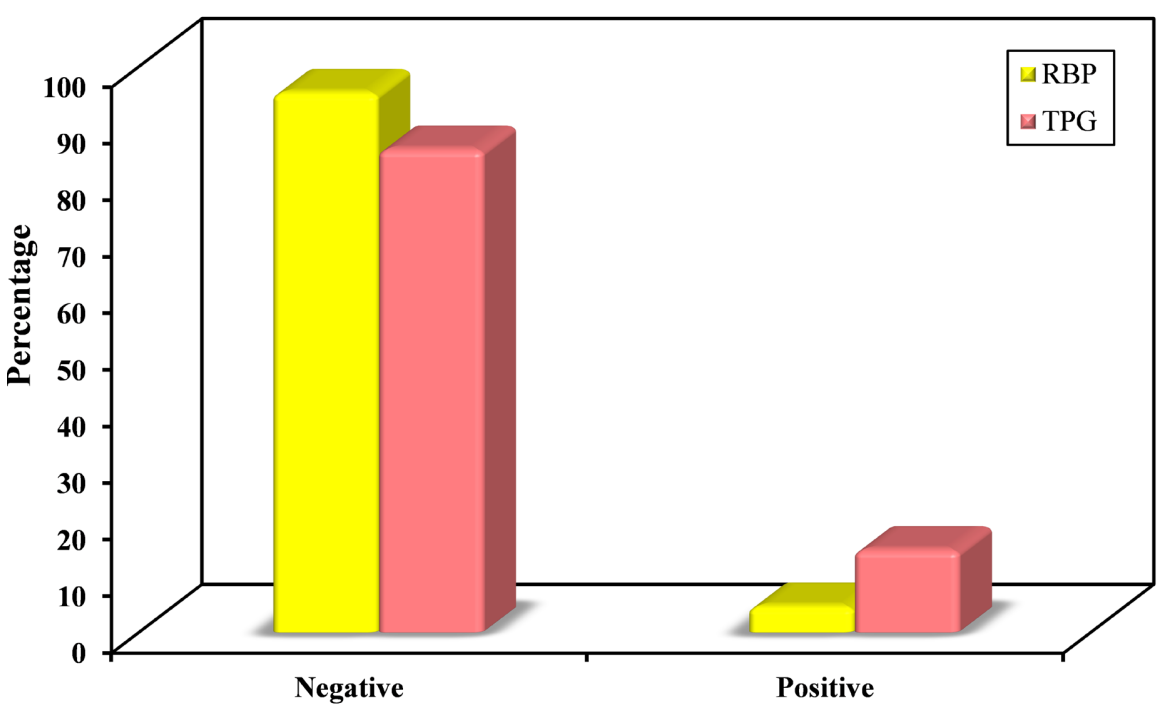

Figure 1. Comparison between random peritoneal biopsy and total peritoneal gutter removal.

49 (37.7\%) cases experienced minimal bleeding, 35 (26.9\%) cases experienced mild bleeding, only one (0.8\%) case suffered from moderate bleeding and 45 (34.6\%) cases had no bleeding. Splenectomy was performed in only one (0.8\%) case. Bowel and ureteric injuries were not encountered (Figure 1).

\section{Discussion}

The removal of the entire parietal peritoneum may be of no benefit if the visceral peritoneum is not removed, but there was a higher incidence of overt and occult disease in parietal peritoneal regions compared to visceral regions, implying that removal of the entire parietal peritoneum may be beneficial. In comparison to parietal regions, the sensitivity of inspection in detecting mild or occult disease was higher in visceral regions. Microscopic pathology was found in the peritoneum close to tumour deposits in $46 \%$ of cases, and in areas without tumour lesions in $34 \%$ of cases (occult disease) [8].

Rosemary Yanick et al. showed that $93 \%$ of ovarian tumors were epithelial and $2 \%$ were mixed groups and stromal tumors and the higher rates were found in patients aged $55-74$ years. $74 \%$ of patients with stage I were under 65 years of age and the median age for all stages was 63 years (Table 1) [9].

In partial agreement with our study, the age ranged from 15 to 90 years with median 50 and we found that $45.4 \%$ of cases were serous carcinoma, $18.5 \%$ were granulosa cell tumor, $13.8 \%$ were mucinous tumor and $12.3 \%$ were endometroid carcinoma. The rest were other epithelial carcinomas and other germ cell tumors.

Artur Czekierdowski et al. stated that M3 and M5 were the most common IOTA $\mathrm{M}$ features reported; they considered that M 3 should suggest high risk of malignancy. Use of simple descriptors rules had sensitivity of $98.4 \%$ and specificity of 63.8\% and ADNEX model had sensitivity of 78\% and specificity of 70\% [10].

In contrast with our study, M 4 was present in most of our cases with a percentage of $72.2 \%$. IOTA ADNEX model revealed that benign in $49.95 \%$ and 
malignant $\%$ in $49 \%$ of cases, the remaining was borderline. It made no difference concerning ADNEX model.

Aditi Bhatt et al. reported that morbidity after total parietal peritonectomy with cytoreductive surgery was $22 \%$. $70 \%$ had one bowel resection and no patient developed bowel fistula or anastomosis leakage. They argued that removing the entire parietal peritoneum had no benefit if the visceral peritoneum was not

Table 1. Sociodemographic and clinical characteristics of the patients.

\begin{tabular}{|c|c|}
\hline Age & Years \\
\hline Min.-Max. & $15-90$ \\
\hline Mean \pm S.D. & $49.97 \pm 13.54$ \\
\hline Median (IQR) & $50(43-59)$ \\
\hline Marital status & No. (\%) \\
\hline Married & $88(67.7 \%)$ \\
\hline Widow & $33(25.4 \%)$ \\
\hline Virgin & $5(3.8 \%)$ \\
\hline Divorced & $4(3.1 \%)$ \\
\hline Family history & No. (\%) \\
\hline No & $110(84.6 \%)$ \\
\hline Ovarian cancer & $11(8.5 \%)$ \\
\hline Breast cancer & $6(4.6 \%)$ \\
\hline Colon cancer & $2(1.5 \%)$ \\
\hline Pancreas cancer & $1(0.8 \%)$ \\
\hline Menopausal state & No. (\%) \\
\hline Perimenopause & $61(46.9 \%)$ \\
\hline Post menopause & $69(53.1 \%)$ \\
\hline Ascites by clinical examination & No. (\%) \\
\hline Absent & $111(85.4 \%)$ \\
\hline Present & $19(41.6 \%)$ \\
\hline Consistency of the lesion & No. (\%) \\
\hline Could not be assessed & $38(29.2 \%)$ \\
\hline Cystic & $40(30.8 \%)$ \\
\hline Semisolid & $31(23.8 \%)$ \\
\hline Solid & $21(16.2 \%)$ \\
\hline Mobility of the lesion & No. (\%) \\
\hline Could not be assessed & $35(26.9 \%)$ \\
\hline Non mobile & $29(22.3 \%)$ \\
\hline Mobile & $66(50.8 \%)$ \\
\hline Surface of the lesion & No. (\%) \\
\hline Could not be assessed & $38(29.2 \%)$ \\
\hline Irregular & $29(22.3 \%)$ \\
\hline Surface & $63(48.5 \%)$ \\
\hline
\end{tabular}


removed. They also concluded that removal of whole parietal peritoneum might be of good benefit when they discovered occult disease (Table 2 and Table 3 ) [11].

Against our study, complications in our cases were not omnius, $0.8 \%$ of cases reported moderate bleeding; the others developed minimal, mild or no bleeding, no case needed blood transfusion. Only one case suffered from splenectomy, no cases suffered from ureteral or bowel injuries. But, total peritoneal gutter removal showed higher detection rate than random peritoneal biopsy.

Andrea Craus Miguel et al. found that compared with peritonectomy, diaphragmatic resection was not associated with higher rates of major complications or long hospital or ICU stay and their results were similar to those in other diaphragmatic peritonectomy versus resection studies (Table 4) [12].

In agreement with our study, complications from modified total gutter removal were not significant and minor complications had been reported as we discussed previously such as bleeding or the need of performing splenectomy.

Table 2. Comparison between random peritoneal biopsy and total peritoneal gutter removal $(\mathrm{n}=130)$.

\begin{tabular}{|c|c|c|c|c|c|}
\hline \multirow{2}{*}{ Positivity } & \multicolumn{2}{|c|}{ Random peritoneal biopsy } & \multicolumn{2}{|c|}{ Total peritoneal gutter removal } & \multirow{2}{*}{$\chi^{2} \quad{ }^{\mathrm{McN}} \mathrm{p}$} \\
\hline & No. & $\%$ & No. & $\%$ & \\
\hline Negative & 124 & 95.4 & 111 & 85.4 & \\
\hline Positive & 6 & 4.6 & 19 & 14.6 & \\
\hline
\end{tabular}

Table 3. Agreement between random peritoneal biopsy and total peritoneal gutter removal $(n=130)$.

\begin{tabular}{|c|c|c|c|c|c|c|}
\hline \multirow{3}{*}{ Random peritoneal biopsy } & \multicolumn{4}{|c|}{ Total peritoneal gutter removal } & \multirow{3}{*}{$x^{2}$} & \multirow{3}{*}{${ }^{\mathrm{FE}} \mathrm{p}$} \\
\hline & \multicolumn{2}{|c|}{ Negative $(n=111)$} & \multicolumn{2}{|c|}{ Positive $(n=19)$} & & \\
\hline & No. & $\%$ & No. & $\%$ & & \\
\hline Negative & 108 & 97.3 & 16 & 84.2 & & \\
\hline Positive & 3 & 2.7 & 3 & 15.8 & 6.311 & 0.04 \\
\hline$\kappa$ (Level of agreement) & \multicolumn{4}{|c|}{$0.183(0.012)$} & & \\
\hline
\end{tabular}

Table 4. Distribution of cases according to complications $(n=130)$.

\begin{tabular}{ccc}
\hline Complication & No. & $\%$ \\
\hline Bleeding & 45 & 34.6 \\
No & 49 & 37.3 \\
Minimal & 35 & 26.9 \\
Mild & 1 & 0.8 \\
Moderate & & \\
Splenectomy & 1 & 0.8 \\
Yes & 129 & 99.2 \\
No & & \\
\hline
\end{tabular}


Sebastian Rufian et al. suggested that serous carcinoma was the most common tumor of epithelial ones followed by endometroid, mucinous, undifferentiated and clear cell carcinomas. They also noted that extended peritonectomy with infra abdominal peritonectomy associated with bilateral flank peritonectomy was done in $12 \%$ of their cases and subtotal peritonectomy was made in $49 \%$ of patients. Also, peritonectomy procedures had a significant impact on the survival of these patients. They also related this procedure to the morbidity and mortality of patients and associated to higher incidence of complications [13].

\section{Conflicts of Interest}

The authors declare no conflicts of interest regarding the publication of this paper.

\section{References}

[1] Kurman, R.J., Carcangiu, M.L., Young, R.H. and Herrington, C.S. (2014) WHO Classification of Tumours of Female Reproductive Organs. $4^{\text {th }}$ Edition, IARC Publications, Lyon.

[2] Fleming, G.F., Seidman, J. and Lengyel, E. (2013) Epithelial Ovarian Cancer. In: Barakat, R.R., Markman, M., Randall, M.E., Eds., Principles and Practice of Gynecologic Oncology, $6^{\text {th }}$ Edition, Lippincott Williams \& Wilkins, Philadelphia, 757-847.

[3] Whitney, C.W. and Spirtos, N. (2009) Gynecologic Oncology Group Surgical Procedures Manual. Gynecologic Oncology Group, Philadelphia.

[4] Bristow, R.E., Chang, J., Ziogas, A., Campos, B., Chavez, L.R. and Anton-Culver, H. (2015) Impact of National Cancer Institute Comprehensive Cancer Centers on Ovarian Cancer Treatment and Survival. Journal of the American College of Surgeons, 220, 940-950. https://doi.org/10.1016/j.jamcollsurg.2015.01.056

[5] Lalwani, N., Shanbhogue, A.K., Vikram, R., Nagar, A., Jagirdar, J. and Prasad, S.R. (2010) Current Update on Borderline Ovarian Neoplasms. AJR American Journal of Roentgenology, 194, 330-336. https://doi.org/10.2214/AJR.09.3936

[6] Morice, P., Uzan, C., Fauvet, R., Gouy, S., Duvillard, P. and Darai, E. (2012) Borderline Ovarian Tumour: Pathological Diagnostic Dilemma and Risk Factors for Invasive or Lethal Recurrence. The Lancet Oncology, 13, e103-e115. https://doi.org/10.1016/S1470-2045(11)70288-1

[7] Silva, E.G., Gershenson, D.M., Malpica, A. and Deavers, M. (2006) The Recurrence and the Overall Survival Rates of Ovarian Serous Borderline Neoplasms with Noninvasive Implants Is Time Dependent. The American Journal of Surgical Pathology, 30, 1367-1371. https://doi.org/10.1097/01.pas.0000213294.81154.95

[8] Sugarbaker, P.H. (1995) Peritonectomy Procedures. Annals of Surgery, 221, 29-42. https://doi.org/10.1097/00000658-199501000-00004

[9] Yancik, R. and Ries, L.G. (1989) Caring for Elderly Cancer Patients. Quality Assurance Considerations. Cancer, 64, 335-341. https://doi.org/10.1002/1097-0142(19890701)64:1+<335::AID-CNCR2820641335>3. $\underline{0 . \mathrm{CO} ; 2-4}$

[10] Van Calster, B., Van Hoorde, K., Froyman, W., et al. (2015) Practical Guidance for Applying the ADNEX Model from the IOTA Group to Discriminate between Different Subtypes of Adnexal Tumors. Facts Views Vis Obgyn, 7, 32-41.

[11] Zanon, C., Clara, R., Chiappino, I., et al. (2004) Cytoreductive Surgery and Intrape- 
ritoneal Chemohyperthermia for Recurrent Peritoneal Carcinomatosis from Ovarian Cancer. World Journal of Surgery, 28, 1040-1045.

https://doi.org/10.1007/s00268-004-7461-x

[12] Querleu, D., Planchamp, F., Chiva, L., et al. (2017) European Society of Gynaecological Oncology (ESGO) Guidelines for Ovarian Cancer Surgery. International Journal of Gynecological Cancer, 27, 1534-1542.

https://doi.org/10.1097/IGC.0000000000001041

[13] Bhatt, A., Kammar, P., Sinukumar, S., et al. (2021) Total Parietal Peritonectomy Can Be Performed with Acceptable Morbidity for Patients with Advanced Ovarian Cancer after Neoadjuvant Chemotherapy: Results from a Prospective Multi-Centric Study. Annals of Surgical Oncology, 28, 1118-1129.

https://doi.org/10.1245/s10434-020-08918-4 